\title{
Task-driven Support Polygon Reshaping for Humanoids
}

\author{
Eiichi Yoshida ${ }^{1 *}$ Oussama Kanoun $^{2}$, Claudia Esteves ${ }^{2}$ and Jean-Paul Laumond ${ }^{2}$ \\ AIST/ISRI-CNRS/STIC Joint French-Japanese Robotics Laboratory (JRL) \\ ${ }^{1}$ Intelligent Systems Research Institute, National Institute \\ of Advanced Industrial Science and Technology (AIST) \\ AIST Central 2, Umezono 1-1-1, Tsukuba, 305-8568 Japan \\ \{e.yoshida\}@aist.go.jp
}

\begin{abstract}
In this paper we address a task-driven motion generation method that allows a humanoid robot to make wholebody motions including support polygon reshaping to achieve the given tasks. In the proposed method, generalized inverse kinematics (IK) is employed with floating-base to generate humanoid whole-body motions that enable the robot to accomplish the tasks according to given priorities. During the motion, several criteria such as manipulability or end-effector position error are tracked. If the desired task cannot be done because of those criteria, a geometric planner for support polygon is activated. Then the whole-body motion is computed again always using the generalized IK solver including stepping motion that realizes the deformed support polygon by using dynamic walking pattern generator. This method provides a way to perform tasks that could not be done without changing the humanoid's support state. We have verified the proposed framework through simulations and experiments using humanoid robot HRP-2.
\end{abstract}

\section{INTRODUCTION AND PROBLEM STATEMENT}

Humanoid robots have made such a progress especially in hardware, now they are expected to execute complicated tasks in environments designed for humans. Their feature resides in the many degrees of freedom as well as the anthropomorphic shape to utilize whole-body motion to achieve tasks.

Recently many studies have been done in generation of whole-body motion to execute tasks such as locomotion, transportation, or service. From a motion planning perspective, most motions are generated in advance based on functional decomposition of the degrees of freedom, for example locomotion and manipulation. Even performing well for combined tasks such as walking while manipulating (e.g., [1]) or digital actor motion coordination (e.g., [2]), such functional decompositions impose some restrictions (for instance, the manipulation task cannot affect to locomotion task). A deeper coordination of all the degrees of freedom should be considered via the generalized inverse kinematics or dynamics (e.g., [3], [4], [5]). However such local methods based on numerical optimization operators do not clarify how to cope with the tasks with different priorities when some of the tasks cannot be achieved.

This paper addresses the following problem: how to recover a positioning error of the humanoid robot body when performing manipulation tasks such as reaching or grasping

\footnotetext{
* His contribution in this research is made at LAAS-CNRS
}

by the end-effector? A solution could be to replan a body re-positioning motion separately in the first stage and then perform the manipulation tasks in the second one. This simple approach would be not natural. The goal here is to generate a whole-body motion including a possible body re-positioning if required. Both re-positioning and manipulation motions should be performed in parallel. The proposed method is based on reshaping the support polygon of the humanoid robot to increase its workarea by coupling generalized inverse kinematics and dynamic walking pattern generator. While using inverse kinematics, the global motion is guaranteed to be dynamically stable. Such a property is a direct consequence of the zero moment point (ZMP) control provided by the pattern generator we use.

Figure 1 illustrates the proposed motion generation framework with an example of a reaching task. Priorities are given to the target task as well as to other tasks such as the position of center of mass. We employ generalized inverse kinematics to generate a whole-body motion for those tasks based on

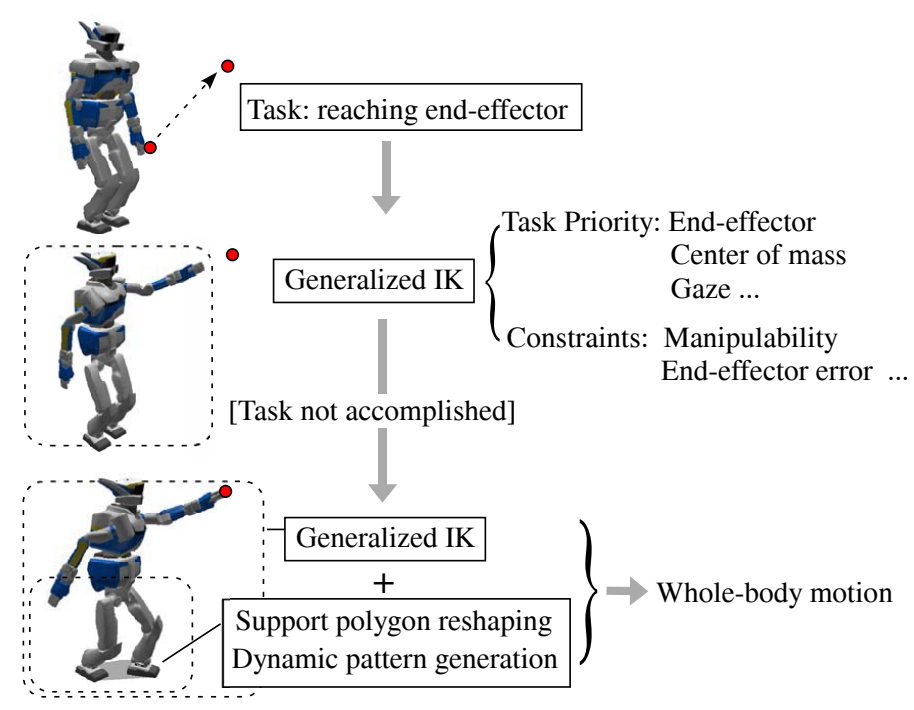

Fig. 1. A general framework for task-driven whole-body motion including support polygon reshaping 
the given priorities [3]. During the motion, several constraints are monitored which are expressed by such measures as manipulability for whole-body, end-effector errors from target, or joint limits.

If the task cannot be achieved because those monitored constraints are not satisfied, a reshaping planner of support polygon is activated automatically to increase accessible space of the robot, keeping the inverse kinematics working to achieve the tasks. The reshaping is performed based on geometric planning to deform the support polygon in the direction required by the specified task. Thanks to the usage of freefloating base, the changes in support phase can be easily integrated in the computation. As a result, the stepping motion is generated using a biped walking pattern generator [6] and the blended whole-body motion including the target task is recalculated.

The effectiveness of the proposed method is verified by the simulations and experiments using humanoid robot HRP-2.

\section{RELATED WORK}

There are many works in the literature that have focused on the generation of whole-body motions for complex mechanisms such as humanoid robots or digital actors. A popular approach for motion specification has been, instead of setting explicitly the value of all degrees of freedom, to only specify the values of a task to be accomplished by a given end-effector. The idea is to benefit from the redundancy of the mechanism to choose the solution that best solves the task according to some constraints. Among these works, inverse kinematics algorithms that project tasks with lower priority into the null space of the Jacobian of the higher priority tasks have been widely studied (e.g., [3], [4], [7], [8]).

As a consequence, different criteria such as manipulability [9], error measurement or singular values of the task Jacobian have been used to monitor overall task feasibility. Most of this works rely on prioritization of the tasks and constraints as in [10], [11] to improve the task achievability.

Another approach for improving the feasibility of a task has been proposed in [12]. Here the degrees of freedom used to solve the task are increased when the goal is unreachable.

Our contribution is to consider the possibility of reshaping the support polygon by stepping to increase the accessible space of the end-effectors in the 3D space. The problem we address can be viewed as a 3D extension of the 2D problem addressed in [1]. In [1] the authors propose a strategy for the control of a pattern generator by monitoring the arm manipulability. While their model lies in the sagittal plane, our approach makes use of the whole body in the 3 directions of the 3D space. Moreover, in spite of our reasoning being based on inverse kinematics and simple geometric support polygon reshaping, our method guarantees that the motion is dynamically stable. This property is a consequence of the pattern generator [6] we use to generate the stepping behavior.

The remainder of this paper is structured as follows: Section III gives an overview of the overall algorithm. In Section IV the way to generate whole-body motions using prioritized IK algorithms and the task feasibility monitoring criteria are described. Section $\mathrm{V}$ presents the geometric method to reshape the support polygon accounting for the task. In Section VI simulation results are shown and discussed.

\section{Method Overview}

We outline the proposed method in this section. The support polygon reshaping integrates two important components, the generalized inverse kinematics and dynamic walking pattern generator. The former provides a general way to deal with the whole-body motion generation to perform the prioritized tasks. The latter takes charge of the stepping motion to change the foot placements.

Figure 2 shows an overview of the method. The task is specified in the workspace as $\dot{\boldsymbol{x}}_{j}$ with priority $j$ from which the generalized IK solver computes the whole-body motion as joint angles $\dot{\boldsymbol{q}}$ of the robot. Meanwhile, several criteria such as manipulability or joint limits are monitored if they do not prevent the desired whole-body motion.

As long as the criteria are satisfied, the computation of whole-body motion continues until the target of the task is achieved. If the task cannot be achieved due to unsatisfied criteria, the support polygon planner is triggered in order to extend reachable space. A geometric module determines the direction and position of the deformation of support polygon so that the incomplete task is fulfilled. The position of a foot is then derived to generate the motion of CoM $\dot{\boldsymbol{x}}_{C o M}$ by using a dynamic walking pattern generator [6].

Using this CoM motion, the original task is then redefined as the whole-body motion including stepping that is recalculated using the same generalized IK solver.

In the following sections, we will describe the generalized inverse kinematics framework and then how the support polygon is reshaped.

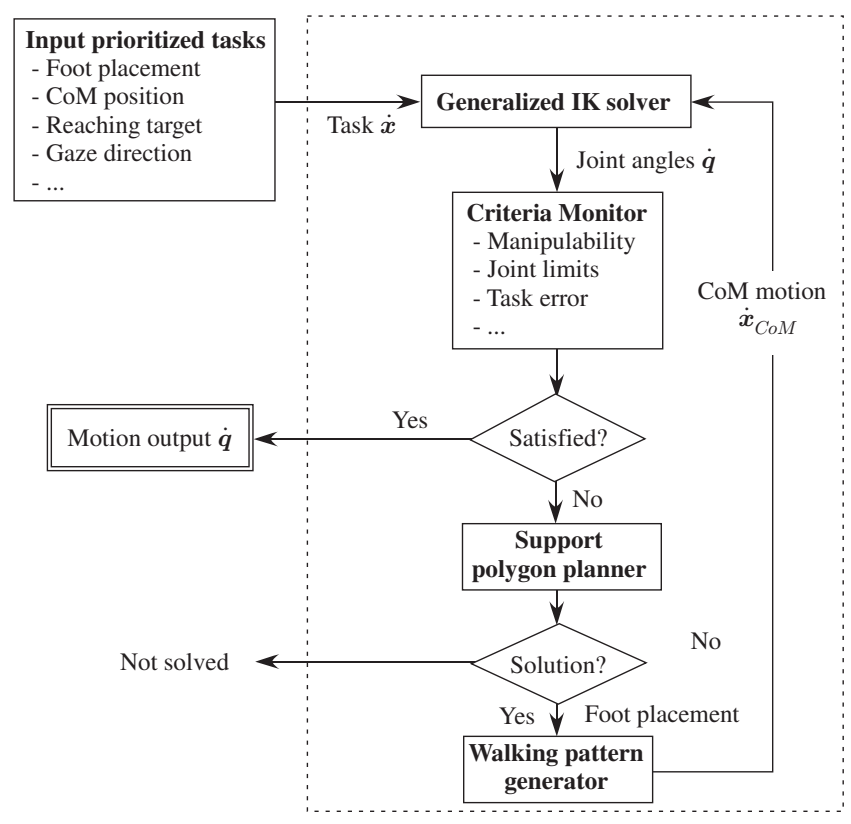

Fig. 2. Method overview 


\section{WHOLE-BODY MOTION GENERATION USING GENERALIZED INVERSE KINEMATICS}

\section{A. Inverse Kinematics for Prioritized Tasks}

As shown earlier, a generalized inverse kinematics is utilized to generate the whole-body motion. The end-effector position is specified as $\dot{\boldsymbol{x}}_{j}$ with priority $j$ in the workspace and the relationship between the joint angle velocity $\dot{\boldsymbol{q}}$ is described using Jacobian matrix, like $\dot{\boldsymbol{x}}_{j}=\boldsymbol{J}_{j} \dot{\boldsymbol{q}}$.

For the tasks with the first priority, using pseudoinverse $\boldsymbol{J}_{1}^{\#}$, the joint angles that achieves the task is given:

$$
\dot{\boldsymbol{q}}_{1}=\boldsymbol{J}_{1}^{\#} \dot{\boldsymbol{x}}_{1}+\left(\boldsymbol{I}_{n}-\boldsymbol{J}_{1}^{\#} \boldsymbol{J}_{1}\right) \boldsymbol{y}_{1}
$$

where $\boldsymbol{y}_{1}, n$ and $\boldsymbol{I}_{n}$ are an arbitrary vector, the number of the joints and identity matrix of dimension $n$ respectively.

For the task with second priority $\boldsymbol{x}_{2}$, the joint angles $\boldsymbol{q}_{2}$ is calculated as follows [3]:

$$
\begin{gathered}
\dot{\boldsymbol{q}}_{2}=\dot{\boldsymbol{q}}_{1}+\hat{\boldsymbol{J}}_{2}^{\#}\left(\dot{\boldsymbol{x}}_{2}-\boldsymbol{J}_{2} \dot{\boldsymbol{q}}_{1}\right)+\left(\boldsymbol{I}_{n}-\boldsymbol{J}_{1}^{\#} \boldsymbol{J}_{1}\right)\left(\boldsymbol{I}_{n}-\hat{\boldsymbol{J}}_{2}^{\#} \hat{\boldsymbol{J}}_{2}\right) \boldsymbol{y}_{2} \\
\text { where } \quad \hat{\boldsymbol{J}}_{2} \equiv \boldsymbol{J}_{2}\left(\boldsymbol{I}_{n}-\boldsymbol{J}_{1}^{\#} \boldsymbol{J}_{1}\right)
\end{gathered}
$$

where $\boldsymbol{y}_{2}$ is an arbitrary vector. It can be extended to the task of $j^{\text {th }}(j \geq 2)$ priority in the following formula [4], [7].

$$
\begin{aligned}
& \dot{\boldsymbol{q}}_{j}=\dot{\boldsymbol{q}}_{j-1}+\hat{\boldsymbol{J}}_{j}^{\#}\left(\dot{\boldsymbol{x}}_{j}-\boldsymbol{J}_{j} \dot{\boldsymbol{q}}_{j-1}\right)+\boldsymbol{N}_{j} \boldsymbol{y}_{j} \\
& \boldsymbol{N}_{j} \equiv \boldsymbol{N}_{j-1}\left(\boldsymbol{I}_{n}-\hat{\boldsymbol{J}}_{j}^{\#} \hat{\boldsymbol{J}}_{j}\right), \hat{\boldsymbol{J}}_{j} \equiv \boldsymbol{J}_{j}\left(\boldsymbol{I}_{n}-\hat{\boldsymbol{J}}_{j-1}^{\#} \hat{\boldsymbol{J}}_{j-1}\right)
\end{aligned}
$$

The CoM Jacobian [13] can be also included as a task.

\section{B. Weighted Pseudoinverse}

In most cases, it is considered to be preferable for a humanoid robot use the lighter links to achieve tasks. For this purpose, we introduce a weighted pseudoinverse:

$$
\boldsymbol{J}_{W}^{\#}=\left(\boldsymbol{J}^{T} \boldsymbol{W} \boldsymbol{J}\right)^{-1} \boldsymbol{J}^{T} \boldsymbol{W}, \boldsymbol{W}=\operatorname{diag}\left\{\sqrt{W_{1}}, \ldots \sqrt{W_{n}}\right\}
$$

The weight $W_{i}$ of each joint is given as the ratio of the mass $m_{i}$ of the link $i$ to the total mass $M$, namely $m_{i} / M$. Moreover, a selection matrix $\boldsymbol{S}=\operatorname{diag}\left\{S_{1}, \ldots S_{n}\right\} \quad\left(S_{i}=\right.$ 0 or 1 ) is multiplied to this inverse to select the activated joints according to the task specification. The selection matrix is set to $\boldsymbol{I}_{n}$ if the all the joints are used to achieve the task.

Using this weighted Jacobian first lighter links are used then heavier ones. By combining a selection matrix $\boldsymbol{S}_{l}$ that forbids using the joints approaching the limit of the movable range, the heuristics of whole-body motion workspace extension [12] can be implemented in a simpler way.

\section{Monitoring Task Execution Criteria}

While the motion is being computed by the generalized IK, several properties are monitored.

One of the important measure is the manipulability [9] defined as:

$$
w \equiv \sqrt{\operatorname{det}\left\{\boldsymbol{J} \boldsymbol{J}^{T}\right\}}
$$

This measure is continuously tracked during the motion generation as well as others such as joint angle limits or end-effector errors from the target. If it becomes below a certain value, it means that it is difficult to achieve the task.

Joint limit constraint can be taken into account by introducing another selection diagonal matrix $\boldsymbol{S}_{l}$ whose $i^{\text {th }}$ components become zero if a joint reaches its limit angle.

As shown in Fig. 2, when the monitor detects the measures that prevent the task from being achieved, the support polygon reshaping is launched to extend the accessible space as detailed in the next section.

\section{SUPPORT POLYGON RESHAPING}

Figure 3 shows the proposed support polygon reshaping scheme. To determine how to reshape the polygon, first the direction angle $a$ between the task target position projected on the ground and the orientation of the closer foot is computed. Then, the foot orientation is set to the closest angle to that of the projected vector within the rotation limits of the foot. The new foot position is chosen in such a way that it maximizes the advance towards the target position. The reshaped support polygon can be then derived from the new foot placement as shown in the right hand side of Fig. 3.

This simple algorithm allows the humanoid robot to make a step motion, keeping a large margin of accessible area for the task by facing the upper body to the target direction.

Then the CoM motion $\dot{\boldsymbol{x}}_{C o M}$ is computed from the new foot position by the walking pattern generator based on preview control of ZMP [6]. The basic idea is to calculate the CoM position and then the leg motions by anticipating the desired future ZMP positions that correspond to the footsteps.

Finally the original task is redefined as another problem of whole-body task using this newly generated CoM motion. The same generalized IK solver framework introduced in IVA is used to incorporate the motion required for task and the stepping motion in the whole-body level.

\section{Simulation and ExPeirmental Results}

The proposed support polygon reshaping method is simulated and experimented to verify its effectiveness using simulator OpenHRP [14] and hardware humanoid platform

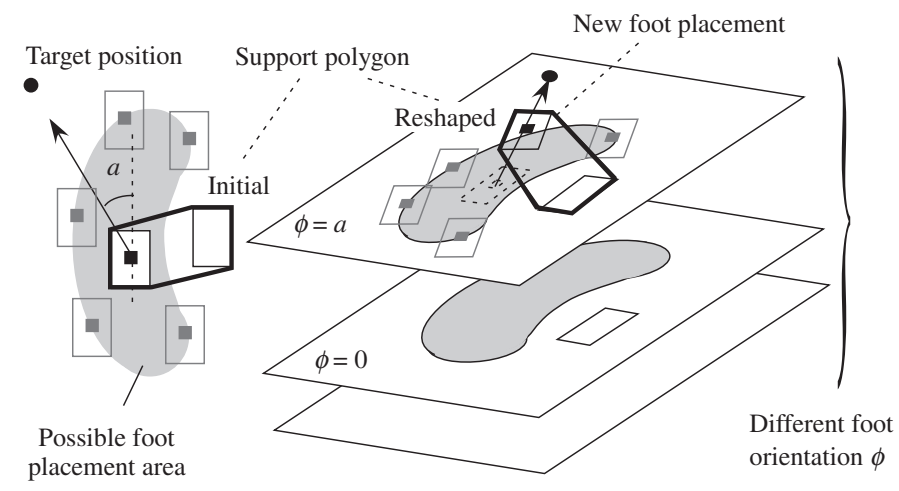

Fig. 3. Support polygon reshaping method 
HRP-2 [15]. The humanoid robot HRP-2 has 30 degrees of freedom with $1.54[\mathrm{~m}]$ in height and $58[\mathrm{~kg}]$ in weight. This robot has two chest joints for pitch and yaw rotation, which extends the capability of whole-body motion.

In the following simulations, the humanoid robot is required to achieve reaching tasks by a hand towards front and side direction. Four tasks are given with the following priority (i) foot placement, (ii) CoM position, (iii) hand reaching task and (iv) gaze direction in the order of higher priority. For all the tasks the weighted Jacobian (4) is utilized for inverse kinematics. As the selection matrix $\boldsymbol{S}$, all the degrees of freedom are used, namely setting $\boldsymbol{S}$ to $\boldsymbol{I}_{n}$, for all the tasks except foot placement that is performed only by the leg joints. The reaching task is defined by the target positions without specifying orientation of the hand.

The monitored criteria here during the motion are the manipulability of the arm and the error between the reference end-effector position and the one calculated by the IK solver. The robot tries to reach the target first with the CoM position at the center of the initial support polygon. If those values go below a certain threshold, the support polygon reshaping process is activated. Here the thresholds of manipulability and end-effector error are empirically set to $1.5 \times 10^{-4}$ and $4.0 \times 10^{-5}[\mathrm{~m}]$ respectively.

We have first conducted simulations for reaching tasks for two different positions of target tasks from the initial position with the parallel foot placement. The front and sideway target positions are $(0.8,0.1,0.8)$ and $(0.4,0.7,0.9)$ respectively in meters, with respect to the global coordinate frame whose origin is located at the center of the feet on the ground, as shown in Fig. 4(a).

Figures 4 and 5 show the movements generated with and without support polygon reshaping for the reaching tasks in the front direction where target position is indicated as a small sphere. We can clearly observe that the task cannot be finished since the arm manipulability goes below the threshold before the hand reaches the target, as the waist of the robot stays almost at the same place to keep its CoM position at the center of polygon. On the other hand, with reshaping the robot can reach its targets using whole-body motion including stepping, which validates the capacity of the proposed method to extend the robot's accessible space. Among the monitored criteria, it was manipulability that goes below the threshold to activate the support in both tasks.

The motion for the second task of reaching sideways is shown in Fig. 6. Similarly as the first task, it cannot be performed without support polygon reshaping due to the arm manipulability that becomes below the threshold before reaching the target. We can see the whole-body reaching motion is mixed with the stepping motion.

Figure 7 shows the manipulability measure of the arm during the forward reaching task illustrated in Fig. 5. Without

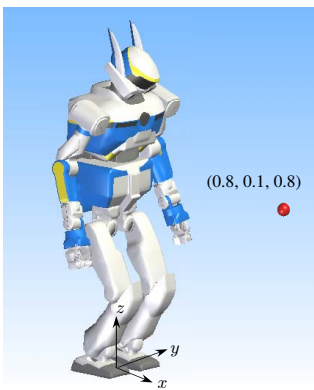

(a)

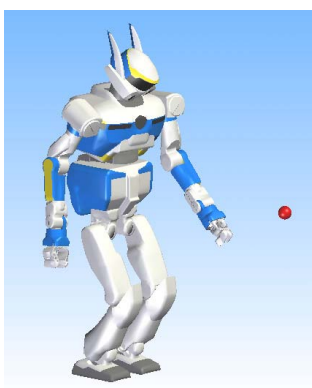

(b)

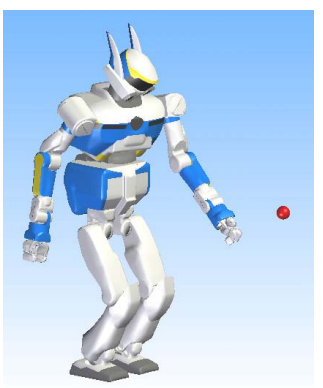

(c)

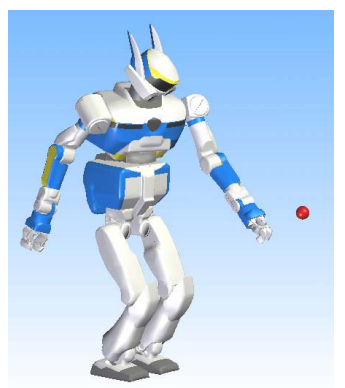

(d)

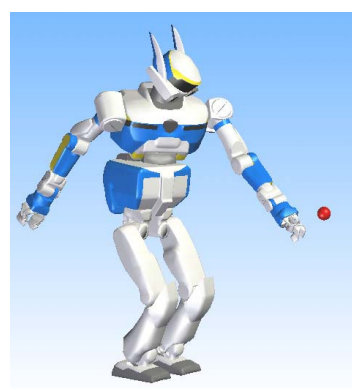

(e)

Fig. 4. Reaching task in the forward direction without support polygon reshaping. The humanoid robot is required to reach the hand toward the target in front (red ball) while keeping the CoM position inside the support polygon. Although the hand approaches to the target, the arm manipulability becomes lower than the threshold defined to avoid the singularity.

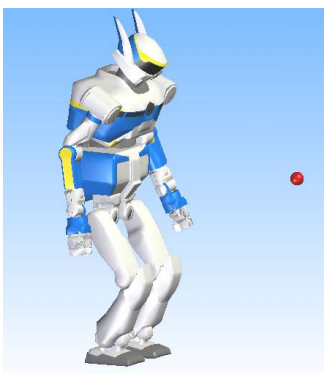

(a)

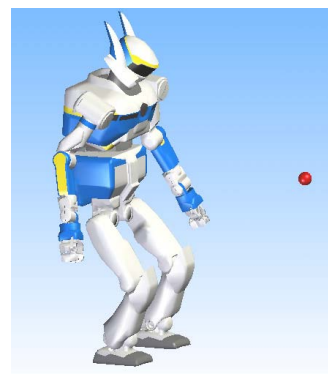

(b)

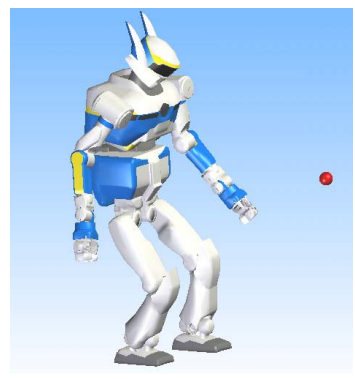

(c)

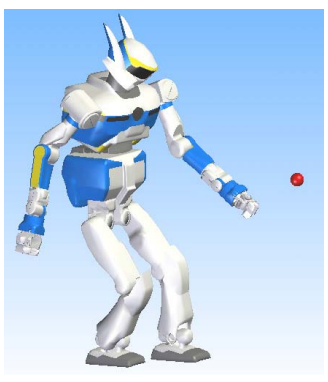

(d)

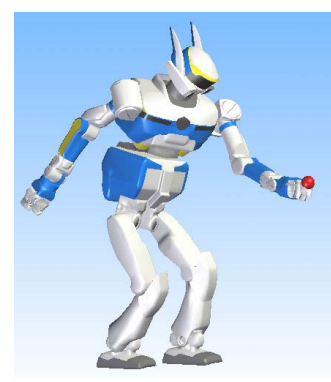

(e)

Fig. 5. Reaching task in the forward direction using support polygon reshaping that determines the placement of the foot for the target point. Using the dynamic walking pattern generator, the position of the CoM is computed to perform the stepping motion including single support phase (b). The whole-body motion is generated using the generalized IK solver and the task is achieved thanks to the extended accessible area provided by the support polygon reshaping. 


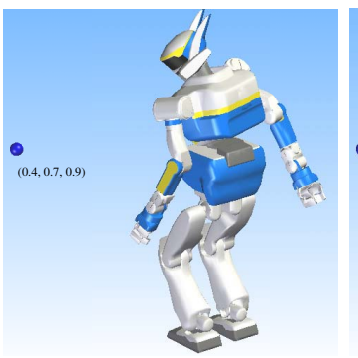

(a)

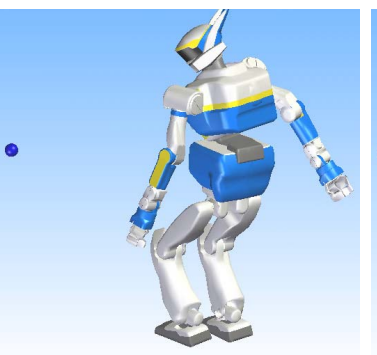

(b)

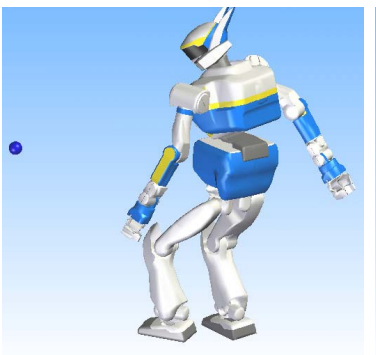

(c)

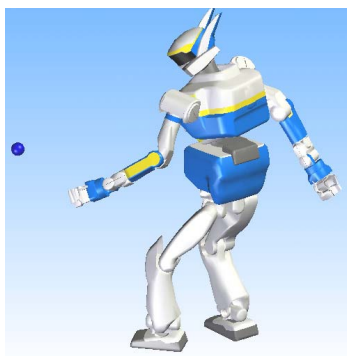

(d)

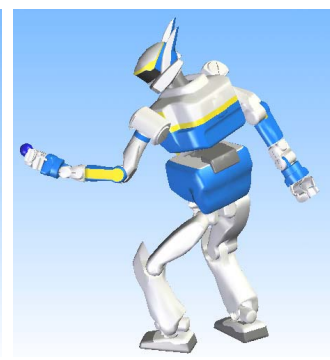

(e)

Fig. 6. Sideways reaching task. The robot first goes to the single support phase (b) and stepping towards the target. After extending support polygon, the robot uses whole-body motion to achieve the required reaching task.

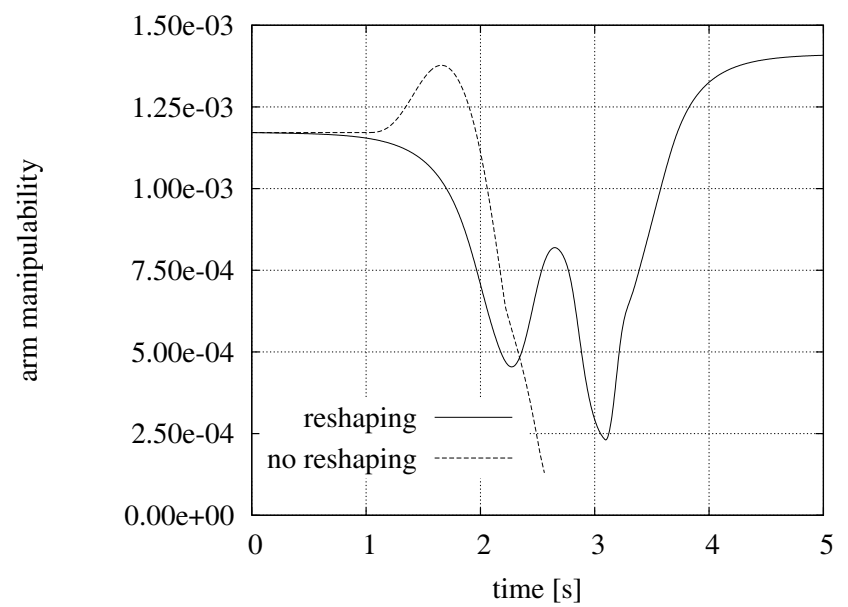

Fig. 7. Manipulability for front reaching. Without support polygon reshaping, the manipulability measure decreases below the threshold. Although it also decreases with reshaping, the manipulability increases in the course of stepping motion.

reshaping, the arm approaches singular configuration where the manipulability becomes lower than the threshold at $2.6[\mathrm{~s}]$ and the computation keeping the same support polygon is discarded. The reshaping starts at this moment to recalculate the overall whole-body motion including stepping. We can see the manipulability regains higher value at the final position.
After verifying the stability of the motion using simulator, we conducted experiments of the generated motion using a humanoid platform HRP-2. Figure 8 shows the snapshots of sideways reaching task. As can be seen clearly in Fig. 8(c), the robot successfully performed the desired reaching task through whole-body motion that unifies reaching task and stepping motion by keeping dynamic balance. Note that the tasks of keeping gaze direction towards the end-effector target position are taken into account in this experiment.

In Figs. 9 and 10, the time development of $x$ and $y$ positions of ZMP measured from the ankle force sensors are plotted for the sideways reaching motion. The dotted and solid lines are the planned and measured trajectories respectively. The shaded areas in those graphs depict the transition of support polygon area projected on $x$ and $y$ axis.

As we can see, the planned trajectories of ZMP always stay inside the support polygon. We can also observe that the measured ZMP values follow the reference value approximately and remain within the support polygon in spite of existence of errors that are supposed to come from force sensor noises. The peaks that are present around 5.8 [sec] are due to the impact generated when the left foot lands on the ground. This impact disturbs the ZMP position during a short period (around 0.2 [sec]), but it does not affect the overall stability.

The movies of simulations are available on our web site ( http://www.laas.fr/ cesteves/humanoids2006/ ).

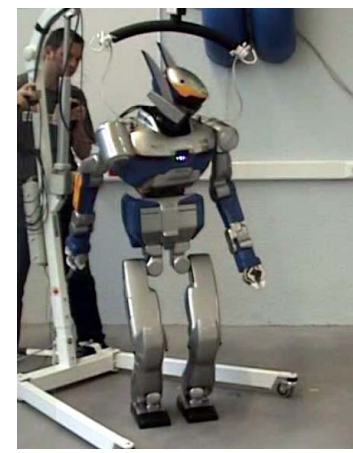

(a)

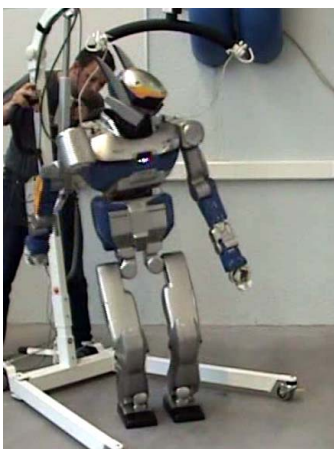

(b)

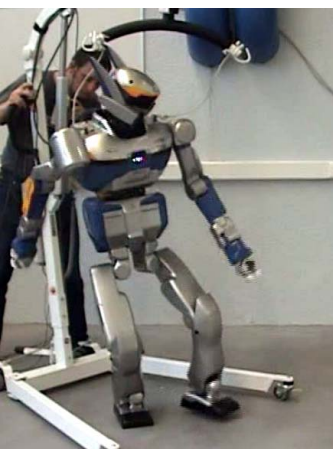

(c)

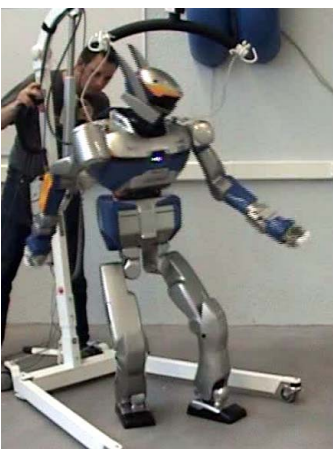

(d)

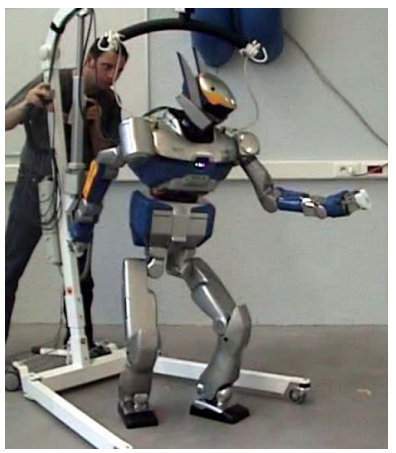

(e)

Fig. 8. Experimentation of sideways reaching task. Putting weight on the right foot in (b), the robot goes through a posture that is not statically stable (c) to finish stepping in (c). The final goal of the end effector is achieved at (e). Notice that the robot makes a whole-body motion including reaching task, stepping and keeping the gaze. 


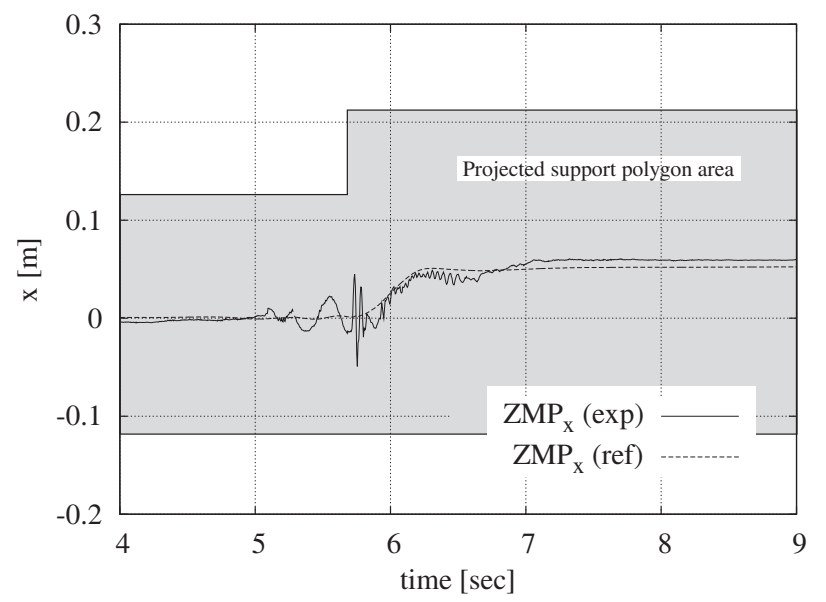

Fig. 9. Experimental and simulation results of transition of $x$ component of ZMP for sideways reaching motion. The shaded area expresses the transition of the projection of support polygon in $x$ axis.

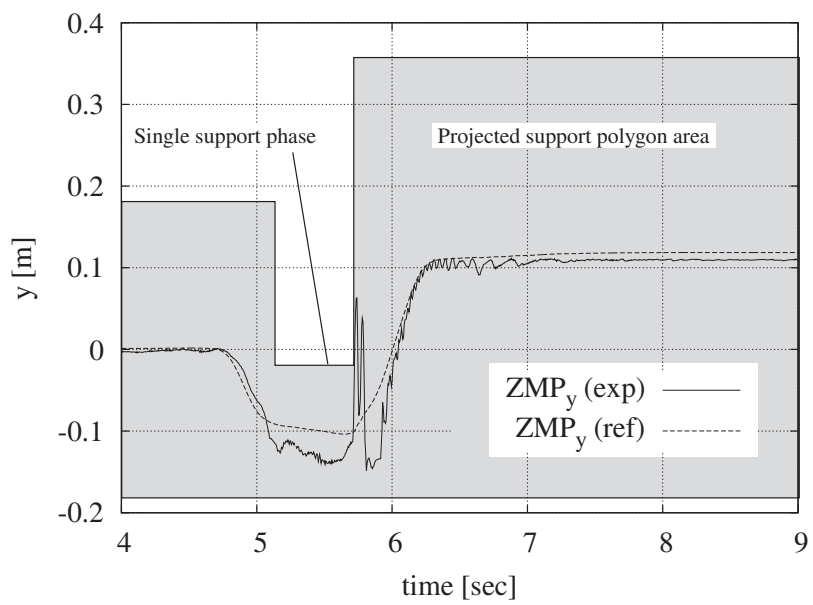

Fig. 10. Experimental and simulation results of transition of $y$ component of ZMP for sideways reaching motion. Similarly in Fig. 9, the shaded area is the projection of support polygon.

\section{CONCLUSION}

In this paper we presented a support polygon reshaping method for whole-body humanoid task execution based on generalized inverse kinematics. Tasks are given to the IK solver to generate whole-body motions by taking account of priorities. Several criteria such as manipulability or joint limits are monitored during the motion generation. If any difficulty is detected in achieving the given task, the support polygon reshaping module is triggered to give a new foot placement that extends the accessible space of the end-effector. A dynamic walking pattern generator provides the stepping motion that is combined with task achieving motion using the same IK solver.

We have performed simulations and experiments of reaching task using humanoid platform HRP-2. The simulation results showed that the proposed method allows the humanoid robot to achieve the tasks that cannot be accomplished due to insufficient manipulability without support polygon reshaping. The feasibility of the generated whole-body motions have been verified by experiments using humanoid robot platform HRP-2 to demonstrate that the robot's accessible space is extended.

Our approach is based on a coupling of inverse kinematics and dynamic pattern generator. The latter guarantees dynamic stability of the whole motion. Future work will include a performance evaluation with respect to another possible approach based on an extension of generalized inverse dynamics [18]. Finally the ultimate goal we pursue is a whole integrated motion planning and control system including gross motion plans [17].

\section{ACKNOWLEDGMENT}

This research was partially supported by the Ministry of Education, Science, Sports and Culture, Grant-in-Aid for Scientific Research (B), 18300070, 2006.

\section{REFERENCES}

[1] H. Yoshida, et al., "Mobile manipulation of humanoid robots - a method of adjusting leg motion for improvement of arm's manipulability," Proc. IEEE/ASME Int. Conf. on Advanced Intelligent Mechatronics, 266-271, 2001.

[2] C. Esteves, et al., "Animation Planning for Virtual Characters Cooperation," ACM Transactions on Graphics, 25-2, 319-339, 2006.

[3] Y. Nakamura, "Advanced Robotics: Redundancy and Optimization," Addison-Wesley Longman Publishing, Boston, 1991.

[4] B. Siciliano and J.-J. E. Slotine, "A general framework for managing multiple tasks in highly redundant robotic systems," Proc. IEEE Int. Conf. on Advanced Robotics, 1211-1216, 1991.

[5] K. Chang and O. Khatib, "Efficient recursive algorithm for the operational space inertia matrix of branching mechanisms," Advanced Robotics, 14-8, 703-715, 2001.

[6] S. Kajita, et al., "Biped Walking Pattern Generation by using Preview Control of Zero-Moment Point," Proc. 2003 IEEE International Conference on Robotics and Automation, 1620-1626, 2003.

[7] P. Baerlocher and R. Boulic, "An inverse kinematics architecture enforcing and arbitrary number of strict priority levels," The Visual Computer, 20, 402-417, 2004.

[8] K. Yamane and Y. Nakamura, "Natural motion animation through constraining and deconstraining at will," IEEE Trans. on Visualization and Computer Graphics, 9-3, 352-360, 2003.

[9] T. Yoshikawa, "Manipulability of Robotic Mechanisms," Int. J. Robotics Research, 4-2, 3-9, 1985.

[10] A. Liegois, "Automatic supervisory control of the configuration and behavior of multibody mechanisms," IEEE Trans. on Systems, Man, and Cybernetics, 7, 868-871, 1977.

[11] L. Sentis and O. Khatib, "A whole-body control framework for humanoids operating in human environments," Proc. IEEE Int. Conf. on Robotics and Automation, 2641-2648, 2006.

[12] Neo. E. Sian, et al., "A Framework for Remote Execution of Whole Body Motions for Humanoid Robots," Proc. IEEE/RAS Int. Conf. on Humanoid Robots, 608-626, 2004.

[13] T. Sugihara, Y. Nakamura and H. Inoue, "Realtime Humanoid Motion Generation through ZMP Manipulation based on Inverted Pendulum Control," Proc. IEEE International Conference on Robotics and Automation, 1404-1409, 2002.

[14] F. Kanehiro, et al., "Virtual humanoid robot platform to develop ", Proc. IEEE/RSJ Int. Conf. on Intelligent Robots and Systems, 1093-1099, 2001.

[15] K. Kaneko, et al., "The Humanoid Robot HRP-2", Proc. IEEE/RSJ Int. Conf. on Robotics and Automation, 1083-1090, 2004.

[16] M. Vukobratović and B. Borovac, "Zero-Moment Point - Thirty-five Years of its Life," Int. J. Humanoid Robotics, 1-1, 157-174, 2004.

[17] E. Yoshida, et al., "Smooth Collision Avoidance: Practical Issues in Dynamic Humanoid Motion," Proc. IEEE/RSJ Int. Conf. on Intelligent Robots and Systems, to appear, 2006.

[18] L. Sentis and O. Khatib, "Synthesis of whole-body behaviors through hierarchical control of behavioral primitives," Int. J. of Humanoid Robotics, 2-4, 505-518, 2005. 